\title{
Skeleton-based Continuous Extrinsic Calibration of Multiple RGB-D Kinect Cameras
}

\author{
Kevin Desai, Balakrishnan Prabhakaran \\ The University of Texas at Dallas \\ Richardson, Texas, USA \\ \{Kevin.Desai,bprabhakaran\}@utdallas.edu
}

\author{
Suraj Raghuraman \\ Mobiweb Inc \\ Plano, Texas, USA \\ suraj@mobiwebinc.com
}

\begin{abstract}
Applications involving 3D scanning and reconstruction \& 3D Teleimmersion provide an immersive experience by capturing a scene using multiple RGB-D cameras, such as Kinect. Prior knowledge of intrinsic calibration of each of the cameras, and extrinsic calibration between cameras, is essential to reconstruct the captured data. The intrinsic calibration for a given camera rarely ever changes, so only needs to be estimated once. However, the extrinsic calibration between cameras can change, even with a small nudge to the camera. Calibration accuracy depends on sensor noise, features used, sampling method, etc., resulting in the need for iterative calibration to achieve good calibration.

In this paper, we introduce a skeleton based approach to calibrate multiple RGB-D Kinect cameras in a closed setup, automatically without any intervention, within a few seconds. The method uses only the person present in the scene to calibrate, removing the need for manually inserting, detecting and extracting other objects like plane, checker-board, sphere, etc. 3D joints of the extracted skeleton are used as correspondence points between cameras, after undergoing accuracy and orientation checks. Temporal, spatial, and motion constraints are applied during the point selection strategy. Our calibration error checking is inexpensive in terms of computational cost and time and hence is continuously run in the background. Automatic re-calibration of the cameras can be performed when the calibration error goes beyond a threshold due to any possible camera movement. Evaluations show that the method can provide fast, accurate and continuous calibration, as long as a human is moving around in the captured scene.
\end{abstract}

\section{CCS CONCEPTS}

- Information systems $\rightarrow$ Multimedia information systems; - Computing methodologies $\rightarrow$ Camera calibration; Reconstruction; 3D imaging; Tracking; Point-based models;

\section{KEYWORDS}

Interactive 3D Tele-Immersion, 3D Calibration, Real-time, 3D Skeleton \& Point-Cloud

Permission to make digital or hard copies of all or part of this work for personal or classroom use is granted without fee provided that copies are not made or distributed for profit or commercial advantage and that copies bear this notice and the full citation on the first page. Copyrights for components of this work owned by others than ACM must be honored. Abstracting with credit is permitted. To copy otherwise, or republish, to post on servers or to redistribute to lists, requires prior specific permission and/or a fee. Request permissions from permissions@acm.org.

MMSys'18, fune 12-15, 2018, Amsterdam, Netherlands

(c) 2018 Association for Computing Machinery.

ACM ISBN 978-1-4503-5192-8/18/06 .. \$15.00

https://doi.org/10.1145/3204949.3204969

\section{ACM Reference Format:}

Kevin Desai, Balakrishnan Prabhakaran and Suraj Raghuraman. 2018. Skeletonbased Continuous Extrinsic Calibration of Multiple RGB-D Kinect Cameras. In MMSys'18: 9th ACM Multimedia Systems Conference, June 12-15, 2018, Amsterdam, Netherlands. ACM, New York, NY, USA, 8 pages. https: //doi.org/10.1145/3204949.3204969

\section{INTRODUCTION}

3D Tele-Immersion (3DTI) systems capture a person, scene, or object, allowing real-time collaboration of geographically distributed users. The availability of commodity RGB-D cameras, such as Microsoft's Kinect [8], has made 3D capture and reconstruction a lot more affordable. Complete 3D capture and live reconstruction of real world objects require the use of multiple such RGB-D cameras. Applications such as collaborative 3DTI $[6,18,23]$, Cyber Physiothery [13], Amphitheater [7], REVERIE - Real and Virtual Come Together in a Virtual Reality [1], Exergames for rehabilitation [9], Penalty soccer game[11], Virtual Laboratories [10], etc. use different number of cameras for capture and reconstruction. Multiple cameras are used in such applications mainly to:

- Generate a complete 3D model

- Provide view dependent 3D rendering

- Perform interactions in the entire 3D space

- Improve visual quality by suppressing the effect of occlusion

For accurate reconstruction, intrinsic calibration of individual cameras and extrinsic calibration between the cameras, both need to be performed. While the intrinsic calibration remains unchanged for a given camera, the extrinsic calibration changes whenever the camera is moved. Various methods exist to calibrate color cameras, both intrinsically and extrinsically. These methods have been extended to work with RGB-D cameras, by calibrating the color and the depth sensors with each other. Most of the existing calibration methods use an external reference object, such as a plane [3], a printed pattern [17], a checker-board [36] or a sphere [33]. Along with that, an expensive, sophisticated robotic system or a person has to be present in the scene to move these objects. Due to the presence of more than one moving objects in the scene, pre-processing needs to be done to extract the correct object being used for calibration. Apart from the fact that this step has high processing overhead, false correspondence points may also be added, leading to erroneous calibration results.

Since these methods need to see the external object clearly in their field of view, cameras being calibrated need to be placed close to each other. Hence, these methods can typically calibrate only two RGB-D sensors at a time. The well-known Checker-board pattern approach gives fairly accurate calibration if performed properly. Regardless of the accuracy, the time taken for calibrating a camera 


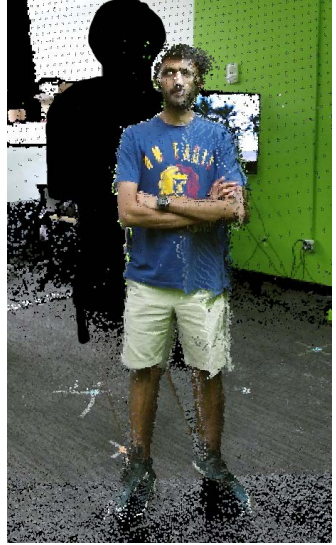

(a)

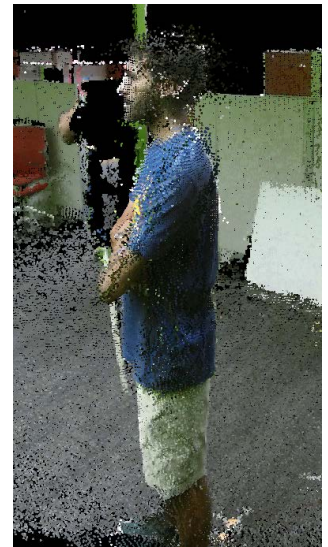

(b)
Figure 1: Reconstructed scene with a person captured with 7 Kinect V2 sensors, and calibrated using skeleton calibration shown from (a) front and (b) side.

pair is at least $10 \mathrm{~min}$. To avoid repeating this time consuming process with all pairs of cameras, calibration propagation strategies are applied. Error in calibration is enhanced during this calibration propagation step. Bundle adjustment techniques are used to reduce error and improve the calibration results. However, this improvement due to bundle adjustment is time intensive and does not take away the fact that the original calibration is inaccurate.

\subsection{Proposed Approach}

In this paper, we propose a skeleton based extrinsic calibration strategy which is extremely fast, accurate and provides continuous calibration. The proposed approach consists of 3 different parts:

- Skeletal joint correspondence: Calibration points are obtained from the extracted Kinect skeleton [30] and are used to simultaneously calibrate multiple cameras. Different constraints are taken into consideration to obtain the best possible correspondence.

- Scene calibration: Transformation matrix calculated from the skeletal joint correspondence is used to calibrate the cameras in a pair-wise manner, using [34].

- Automatic re-calibration: Calibration error is continuously checked in the background so as to perform automatic re-calibration in real time.

The proposed skeleton calibration method is able to accurately calibrate a scene with 7 Kinect V2 sensors in under a minute, without any manual intervention or external reference object, except for person moving around in the acting space. Figure 1 shows a reconstructed 3D model, by transforming all 7 point clouds using the calibration matrices provided by our method.

\subsection{Contributions}

The main contributions of our work are:

- An automated skeleton calibration strategy is proposed that does not require any manual or external intervention. It removes correspondence errors occurring due to false capture of the reference object.
- A robust skeleton-based point acquisition strategy is used for calibration. Since the proposed method does not depend on color at all, it eliminates the error propagation from color and depth sensor calibration.

- Since all the cameras are able to see the same person and extract the skeleton, the calibration method is performed simultaneously for all camera pairs by using the acquired joint correspondence.

- Real-time tracking of calibration errors and automatic recalibration of the cameras is performed.

\section{RELATED WORK}

Calibration is the fundamental and the foremost step for capturing large environments and placing them in the same global coordinate space. Camera misalignment errors have a significant effect on the accuracy of the $3 \mathrm{D}$ reconstruction as well as on the efficiency of the systems. This effect has been studied in detail in [12]. A lot of research has been done in the field of camera calibration over the years. Majority of the work is done in color camera calibration. With the advent of RGB-D cameras such as Microsoft's Kinect [8], the topic of depth camera calibration has gained a lot of research interest. Our primary focus is to calibrate RGB-D cameras, especially the widely used Kinect. Hence, in this section we are narrowing down on only those works which use Kinect for depth camera calibration.

A checkerboard pattern based calibration was introduced by [36]. The checkerboard pattern is printed on a plane and shown to the cameras. The method uses points at the corners of the white and black squares, to identify point correspondence. For calibrating the Kinect, either the infrared image is used, or the color cameras are calibrated with each other, and the depth cameras are calibrated with the color cameras using the infrared image. Due to the various cross calibrations involved, the process is time consuming and susceptible to inaccurate calibration, requiring re-calibration at various steps. An extra overhead Kinect was used by [22] to minimize the propagation error, by calibrating all the other Kinect sensors with the overhead Kinect. OpenPTrack visual tool was provided by [25], which calibrates multiple cameras by showing the checker-board progressively to all the cameras.

An intersection of plane based calibration method was proposed by [3]. A plane whiteboard is shown to the camera, and rotated in an orthogonal manner on all three axes. The plane is tracked using a color detector. The points on the plane are fit to a plane model, and the intersection of these planes is used as the reference point between cameras. This is a very time consuming process, requiring accurate color to depth calibration and plane rotation. The result depends on the angles between the cameras and the real world board plane used. Methods based on the corners of the board $[4,15,19]$ have also been proposed, but are influenced by edge noise, leading to poor calibration.

A 3D reconstruction approach based on block patterns was proposed by [17]. The block pattern is printed and shown to the cameras. The approach can calibrate multiple Kinect V2 cameras, by calibrating their individual color cameras. The method may have inaccuracies due to cross calibration between color and depth. Iterative Closest Points (ICP) is used to refine the results. While this method takes less time, the calibration is not very accurate. 


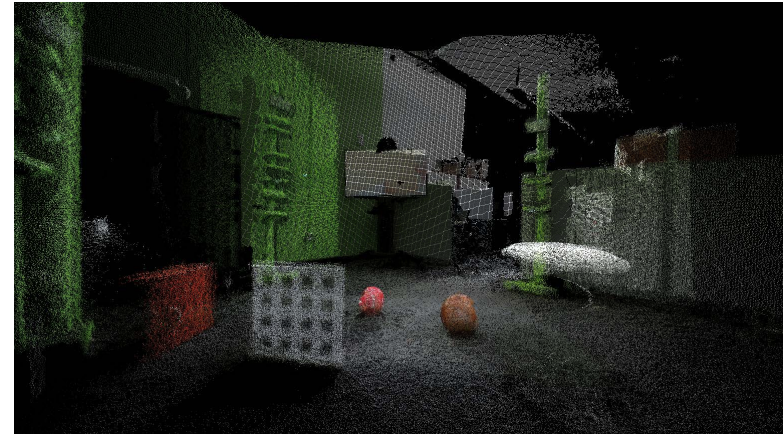

(a)

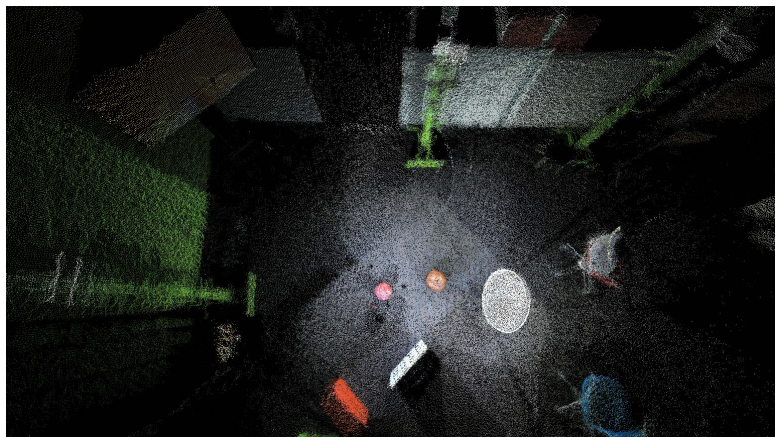

(b)

Figure 2: Point cloud rendering of a scene with multiple objects, captured using 7 Kinect V2 sensors, calibrated simultaneously using our skeleton calibration method with (a) front view and (b) top view.

A color to depth calibration for Kinect was proposed by [31]. A spherical object is shown and detected on both, color and depth images. An ellipse is used for tracking in color image, and a Random Sample Consensus (RANSAC) detector is used in the depth image. The centers of circle (depth) and ellipse (color) are used for calibration. This method was shown to be very effective in calibrating color and depth cameras of the Kinect. However, this method cannot directly be applied to multiple Kinect calibration, due to its planar point selection scheme.

Use of a spherical object center for extrinsic calibration of multiple RGB-D cameras is proposed in [28]. Manual effort is required in removing outliers and hence the method is not robust. Also, the cameras are not time synchronized which make the technique labor intensive as the sphere needs to be physically moved to different locations while calibrating. [33] removes the labor intensiveness by time synchronizing the cameras in the network. The sphere ball is painted yellow to easily detect it and is attached to a pipe which is moved around in the scene by a person. The method seems to calibrate the cameras fairly quickly with very low error rate. But, there maybe occlusion as well as a lot of false positives in detecting sphere in the depth frame due to the fact that human head is almost spherical. Also, the method is still invasive in terms of using a sphere - controlled by a human or a robot - as a reference object.

\section{SKELETON CALIBRATION}

Calibration of multiple Kinect cameras using our skeleton calibration method goes through the following steps:

- Skeletal Joint Correspondence is obtained between the cameras using skeleton as the reference object making the method non-invasive and at the same time robust to errors induced by external objects. It is made sure that the cameras being calibrated all see the same orientation of the person's extracted skeleton. Motion, spatial and temporal constraints are added after checking the accuracy of the skeleton correspondence joints.

- Scene Calibration is performed on a pair by pair basis [34], using the above skeletal joint correspondence.

- Automatic Re-calibration can be performed without disturbing the application or any manual intervention. Calibration error is calculated on the fly by comparing the calibrated skeleton joints. If any of the cameras are moved, the calibration error will go above a certain threshold and the method will automatically re-calibrate the cameras.

Figure 2 shows point cloud rendering for 7 Kinect V2 cameras calibrated using our method, from front and top views. The setup contains multiple objects distributed across the scene to visually check for calibration errors.

\section{SKELETAL JOINT CORRESPONDENCE}

Skeleton Calibration uses the 3D joint positions of the extracted user skeleton as point correspondences between cameras. In order to automatically identify and select these points, the following things need to happen:

- Appropriate joints needs to be selected as probable correspondence points. The skeleton needs to be accurately detected by all cameras such that the left and right joints are labeled consistently.

- Constraints need to be applied so as to have wide-spread and correct point correspondences.

\subsection{Joint Selection}

At a given instant, each Kinect V2 camera captures 25 joints that make up the skeleton [30]. In order to obtain proper point correspondence between cameras, only those joints which are visible by both of them are taken into consideration. The depth image captured by the Kinect V2 contains a lot of salt and pepper noise especially around object edges. Since the skeleton is extracted from the depth image [30], the noise sometimes leads to inaccurate skeleton joint estimation, especially for the end joints. To make sure the joints used are accurate, we remove the end joints and take only 16 out of the 25 joints into consideration for being correspondence joints.

All the 16 joints need to be tracked correctly to obtain proper calibration. Each joint given by Kinect V2 has a state associated with itself which denotes if it is Tracked, Not-Tracked or Inferred. Joints which have the state Tracked in both cameras are taken as acceptable correspondence points. Each joint which is an acceptable correspondence point is stored in corresponding arrays. In order to maintain a proper spread of points all over the scene, the person is asked to move around in the scene freely. However, this does not 


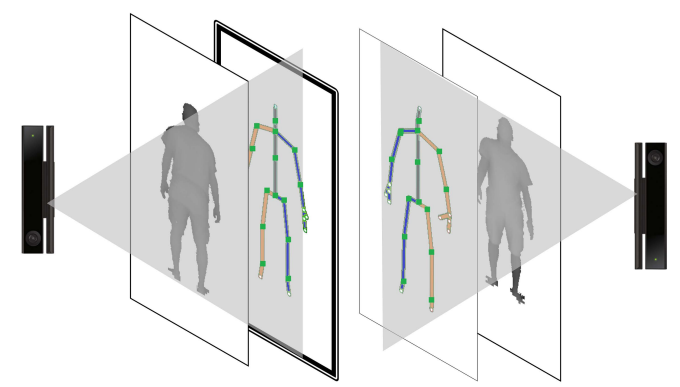

Figure 3: Skeleton captured by 2 cameras facing each other.

control how calibration is performed for the given skeletal frame which may consist of multiple correspondence points. To cover all the points in a widespread region, a specific pattern is adopted by realigning the correspondence points' arrays. This realignment is a one-time static step which assumes the general skeleton structure and randomizes it based on the position of joints in the body.

Accurate skeletal joint correspondence is obtained by making sure that the left and right joints for the skeleton are labeled consistently by all the cameras. As long as a skeletal figure is detected, it is extracted and the joints are tagged as left or right based on what is visible to the Kinect. Figure 3 shows a setup where two Kinect cameras being calibrated are placed facing each other with the person in the center. As shown, both the cameras are able to detect a skeletal figure and tag it left and right based on what each of them sees. The camera on the left tags the skeleton correctly - right side of the skeleton is tagged right (blue) and left side of the skeleton is tagged left (orange). The camera on the right tags the skeleton incorrectly - right side of the skeleton is tagged as left (orange) and left side of the skeleton is tagged right (green). We take the joint $3 \mathrm{D}$ positions directly as correspondence points to calibrate based on the tag associated with it. In such cases, if we were to select the left-shoulder joint, the left camera would take the 3D point on the left whereas the right camera would select the $3 \mathrm{D}$ point on the right. This would result in incorrect point correspondence and hence incorrect calibration. To avoid or solve such situations, we need to flip the skeleton tags.

Skeleton flipping is performed by first detecting the direction of the person. There exist several Kinect based methods, such as $[5,24,26]$, that determine the frontal view of the person with or without using OpenCV algorithms. In our system, we use a strategy similar to [26], modified for use with more than 2 cameras, to obtain the person direction. Once the direction of the person is known, we check if the camera detected direction is correct or not. If not, we have to flip the tags for left and right joints. To do so, we take a copy of the original joint assignments. We copy the joints that are tagged right, from the original skeleton to the corresponding left joints in the new skeleton copy. We do the same for the original left joints. Using the mentioned flipping approach, we determine the direction of the person accurately and orient all the individual skeletons in the same direction so as to have correct skeletal joint correspondence. After selecting the correspondence joints, all of them are checked for the selection constraints.

\subsection{Selection Constraints}

Adopting the above strategies covers the major selection criteria for skeletal joint correspondence. But not all frames captured by the Kinect can be directly used for calibration. Temporal, motion and spatial constraints are applied to ensure that the joints selected are effective to represent the point correspondences.

Temporal Constraint: The data captured by the Kinect V2 sensor is at $30 \mathrm{fps}$. Since the Kinect V2 sensor does not allow triggered image capture, it is ensured that the images are at most one frame apart (approximately $30 \mathrm{~ms}$ ). All the Kinect V2 cameras are connected to individual computers connected with each other over the network. In order to have proper temporal correspondence between the camera frames, all the computers are synchronized using the Network Time Protocol (NTP) and are millisecond precise. To avoid small synchronization errors, a minimum of $\omega$ time is kept between two skeleton frame captures.

Motion Constraint: Since there is no real way of regulating when an image is captured by the Kinect, even a small motion can result in different positions of the skeleton within a millisecond. Using skeleton joints, captured while the person is moving, would result in non corresponding points being used for calibration; this would eventually lead to inaccurate calibration. So, a motion constraint is applied to ensure that the points selected remain the same (motion less than $5 \mathrm{~mm}$ ), for at least a couple of frames, before they can be used for calibration.

Spatial Constraint: Points from all parts of the scene need to be captured to have accurate calibration. If all points are captured based only on the motion constraint, then a large number of points would belong to the same region of the scene. To get representative points from the entire scene, and to avoid oversampling a region, a spatial constraint is enforced. The spatial constraint ensures that there is at least $\delta$ distance between two consecutive skeleton capture for calibration.

\section{SCENE CALIBRATION}

The complete scene is calibrated in a pair-wise manner. Each camera is paired up with all the other cameras. At each camera, the point is selected using the above procedure, and is time stamped. Once the number of points crosses the threshold $\eta$, registration is performed using the points. Subsequently, registration is performed after every $\eta$ new points are added, until the transformation matrix is stable.

The collected point pairs, for each pair of cameras, are registered with each other. Registering them aligns the two camera views with each other, to generate a single world coordinate system having accurate calibration. The registration problem can be stated as given, two m-dimensional point sequences $P=\left\{p_{i}\right\}$ and $Q=\left\{q_{i}\right\}$, where $i=1,2, \cdots, n$; then determine the rotation matrix $R \in \mathbb{R}^{m \times m}$, translation vector $t \in \mathbb{R}^{m}$ and scalar $c$, such that the average of the squared errors, $E(R, t, c)$, is minimized.

$$
\underset{R, t, c}{\operatorname{argmin}} E(R, t, c)=\underset{R, t, c}{\operatorname{argmin}} \frac{1}{n} \sum_{i=1}^{n}\left\|p_{i}-\left(c R q_{i}+t\right)\right\|^{2}
$$

There are many closed form solutions to the registration problem $[2,16,34]$. Popular methods $[2,16]$ are known to provide incorrect $R$, for highly noisy point sets compared to [34]. The likelihood of having corrupted point data is minimal, due to our use of multiple skeletal joints as correspondence points. Hence, we use the solution proposed by [34] to register the point sets. The approach first makes the points translation independent, by subtracting the centroid from 


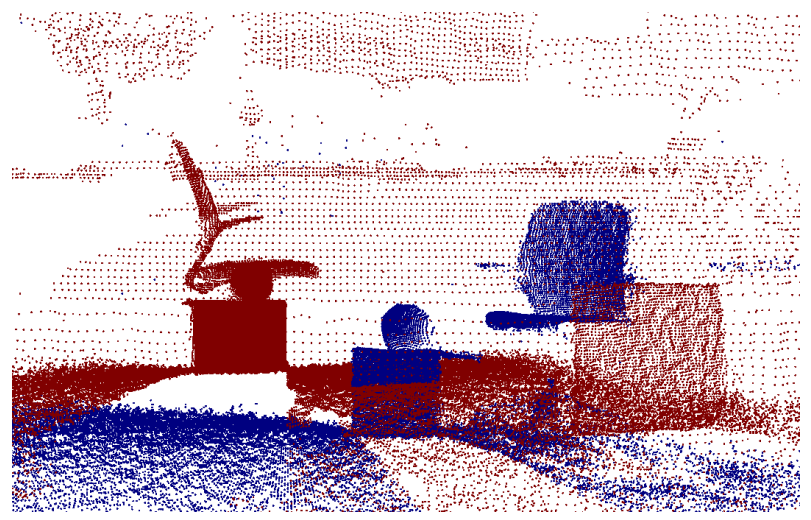

(a)

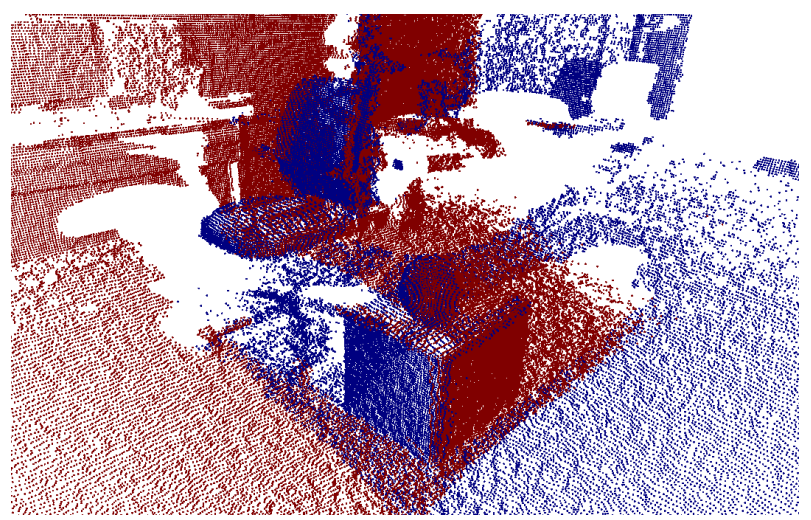

(b)

Figure 4: Calibration results between Kinect A (red) and Kinect B (blue), (a) before calibration and (b) after calibration.

all the points. Then SVD is performed on the covariance matrix of the translation independent points. The reflective artifact is corrected using the determinant of unitary matrices from SVD, and the $R$ is estimated. The scalar $c$ is estimated using the singular values. The translation $t$ is determined by transforming the centroid to the new orientation, and calculating the difference in position. This approach provides very good alignment, even with as little as 10 good skeletal frames, about 100 correspondence points, check Figure 4.

\section{AUTOMATIC RE-CALIBRATION}

Re-calibration of the cameras is an important benefit of our skeleton calibration method. It is not unusual for someone to pass near the camera and move them out of alignment when the system is being used after calibration. A main aspect of re-calibration is that it can be done automatically as long as a human is present in the scene. The application does not have to be disturbed by introducing an "invasive" object in the scene - such as a ball or checker board. All the individual Kinect skeletons are calibrated using the calibration results. The calibration error is calculated by finding the maximum distance between these calibrated skeleton joints. Since all the skeleton joints are tagged appropriately, we use the tracked joints from each camera pair and find the Euclidean distance between them. The maximum distance of the joints is taken as the calibration error for the corresponding camera set. This process takes very little time and processing power and can be run in the background. A threshold for the calibration error is set to check if any of the cameras have moved. If the calibration error goes beyond the threshold, then our skeleton calibration method runs automatically providing back the proper alignment.

Algorithm 1 summarizes the proposed approach as pseudocode.

\section{EVALUATION \& DISCUSSION}

\subsection{Experimental Setup}

The experimental setup consisted of 7 Kinect V2 sensors positioned to capture a $4 m \times 3 m$ area. The sensors were arranged facing each other with a maximum of $2 m$ of height variation. Each sensor was connected to an individual machine, having an Intel Xeon $3.0 \mathrm{ghz}$

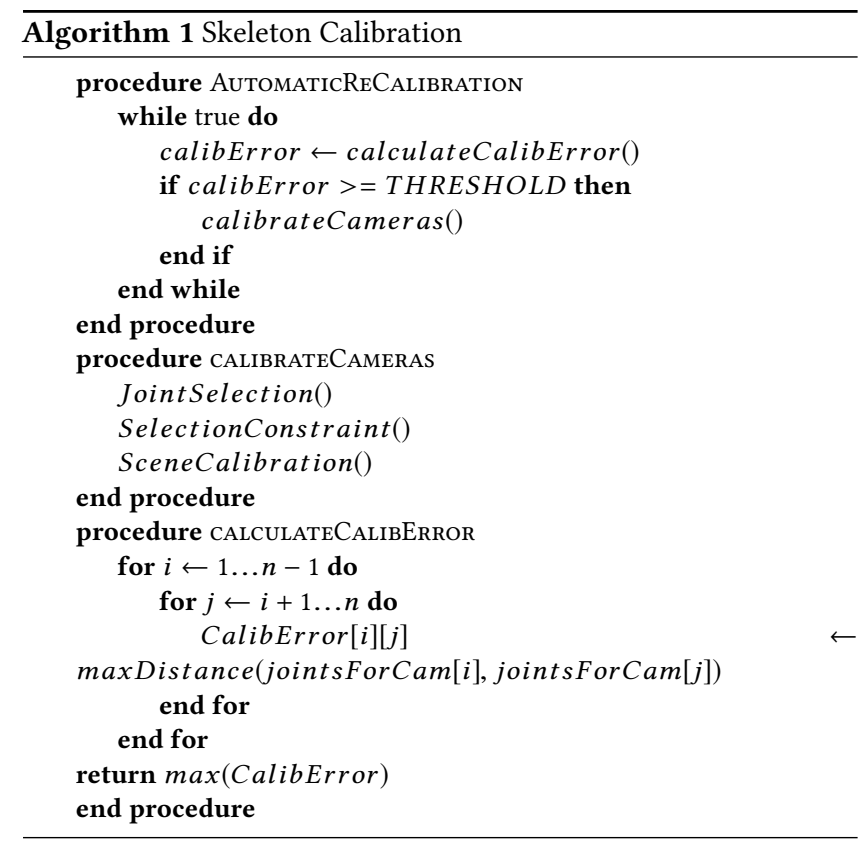

processor, 12GB RAM, and Nvidia Quadro 4000 graphics. The machines were connected to each other on a 1 Gbps network. The system clocks are synchronized within $\pm 5 m s$ of each other, using NTP. The approach is implemented in $\mathrm{C}++$, with GPU optimization on Windows. To obtain about 20 frames in a second as well as to ensure synchronized skeleton capture, the temporal constraint $\omega$ value was set to $50 \mathrm{~ms}$. In order to cover a wide scene space for calibration and at the same time perform quick calibration, the spatial constraint minimum distance $\delta$ value was set to $20 \mathrm{~cm}$. To ensure fast calibration of the cameras, re-calculation of the transformation matrix was done after every 20 frames, setting $\eta$ to 20 .

\subsection{Compared Methods}

Our skeleton calibration method was compared against three prominent methods, representing each style of extrinsic calibration for 


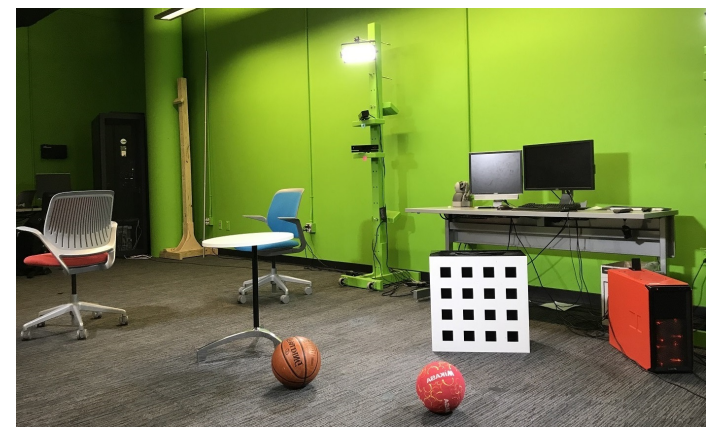

Figure 5: RGB image of the calibration scene setup.

Kinect sensors. For this comparison, to avoid calibration propagation errors, only three Kinect V2 sensors were used. The sensors were positioned in an L shape, with each of them located at a corner and with some height variation between them. Since bundle adjustment methods mask the underlying deficiencies in the original methods, we perform our comparison without using any calibration propagation strategy. Hence, the sensors were arranged such that they have some overlap between them allowing all methods to get point correspondence in every frame. All sensors were calibrated with respect to the center sensor and the result rendered as a point cloud. Figure 5 shows the RGB image of the 3 Kinect camera setup used for evaluation, with different objects present in the scene.

Checker-board pattern proposed in [36] was printed and stuck on a board. The checker squares were of size $5 \mathrm{~cm} \times 5 \mathrm{~cm}$ and the entire pattern was a grid layout of $10 \times 10$. About 50 frames were captured per camera set. The Matlab code for this method was run on the captured data to obtain the pairwise calibration matrices.

Plane intersection approach proposed in [3] required us to use a white board plane of size $100 \mathrm{~cm} \times 80 \mathrm{~cm}$. On an average, 80 orthogonal frames were captured per camera set, which amounts to about 250 correspondence points. These process was repeated for all cameras to obtain the calibration results for this method.

Visual pattern approach [17] calibrated the camera set using the proposed tool. A fixed size pattern proposed in the paper was printed on a A4 sheet. The tool calculates the calibration matrices on the fly and keeps update the visual results. They provide ICP option in the tool which was applied on their results to provide the calibration results.

Our method was run to capture around 150 correspondence points, so as to have a fair comparison.

\subsection{Performance Analysis}

Current methods that exist to compare camera calibration results, such as [29,32], need ground truth data of the scene. They either use existing LIDAR data or manually annotated data as the ground truth. [32] calculates the accuracy and completeness scores to evaluate their result against the ground truth. Since we don't have any ground truth information, we can not use the above approaches. The skeleton captured by the Kinect is fairly accurate, with error range of $1-10 \mathrm{~mm}$ in normal body posture (standing and walking). However, the error quickly increases to about $5 \mathrm{~cm}$ in poses such as sitting, lying down, crouching, etc [20,27]. Hence, in our calibration setup, the person has to be in a normal pose (standing or walking).
The calibration quality of our skeleton calibration method was evaluated on the following fronts:

- Visual Quality Analysis

- Real-time Performance

- Calibration Error Analysis

Table 1 shows the comparison of the methods in terms of time taken and calibration errors. Based on the results, our method performed significantly better on all fronts.

Visual quality of the calibrated setup using the 4 different methods can be seen in the Figure 6. It shows the visual error in the calibrated result with different color markings. Apart from our skeleton calibration method, all other methods had significant calibration errors which can be clearly seen in the misalignment of the different objects in the scene - orange basketball, red volleyball, chess pattern, CPU, chairs, white coffee table, pillars on the side. There is also an error visible near the red volleyball for our method as well. But the misalignment error is very minimal and hard to notice in normal conditions.

Time taken by our method was the minimum, requiring less than a minute to calibrate all 3 sensors. The live scan 3D visual pattern method [17] took about 2 to 3 minutes for all cameras. Both the methods, [3] and [36], required multiple attempts to calibrate taking over 30mins to calibrate, with most of the time spent moving the boards, or re-calibrating to get better accuracy.

Calibration errors for our method, both maximum and average Euclidean distance errors are the lowest $-3.21 \mathrm{~cm}$ and $1.47 \mathrm{~cm}$ respectively. After various attempts of re-calibration, the best result that we could achieve for checkerboard method [36] was off by a max of $14.54 \mathrm{~cm}$ and an average of $8.45 \mathrm{~cm}$. Plane intersection approach of [3] had a max error rate of $9.12 \mathrm{~cm}$ and an average error rate of $4.90 \mathrm{~cm}$. Visual pattern method [17] had a max error of $6.85 \mathrm{~cm}$ and an average error of $4.12 \mathrm{~cm}$, despite performing ICP to refine the results.

\subsection{Re-calibration Evaluation}

Video showing the proposed continuous calibration approach for 7 Kinect V2 cameras can be found at - https://youtu.be/8N1ob3dDxpY

We set the calibration error threshold for re-calibration to be $5 \mathrm{~cm}$. If there is a nudge in any of the camera set and if it results in calibration errors greater than the threshold then re-calibration will happen automatically to fix this. In the case of the example shown in the accompanying video, the error because of the camera movement was $6.4 \mathrm{~cm}$. The automatic re-calibration strategy fixed this by bringing the error down to $3 \mathrm{~cm}$. It took about $3 \mathrm{~s}$ to perform the re-calibration and get the error below the threshold.

Table 1: Calibration comparison in terms of Max and Average Error for $\mathbf{5 0 0}$ frames and approximate Time taken to calibrate 3 Kinect cameras.

\begin{tabular}{|l|l|l|l|}
\hline Calibration Method & $\begin{array}{l}\text { Time } \\
(\mathbf{m i n})\end{array}$ & $\begin{array}{l}\text { Max } \\
\text { Error } \\
(\mathbf{c m})\end{array}$ & $\begin{array}{l}\text { Avg } \\
\text { Error } \\
(\mathbf{c m})\end{array}$ \\
\hline Checker-board [36] & 40 & 14.54 & 8.45 \\
\hline Plane Intersection [3] & 35 & 9.12 & 4.90 \\
\hline Visual Pattern [17] & 3 & 6.85 & 4.12 \\
\hline Our Method & $\mathbf{1}$ & $\mathbf{3 . 2 1}$ & $\mathbf{1 . 4 7}$ \\
\hline
\end{tabular}




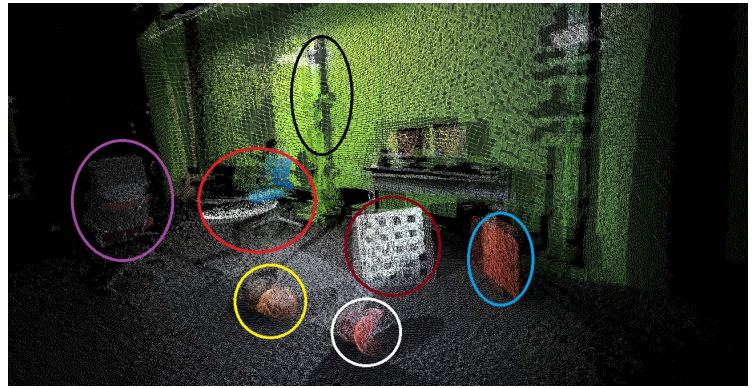

(a)

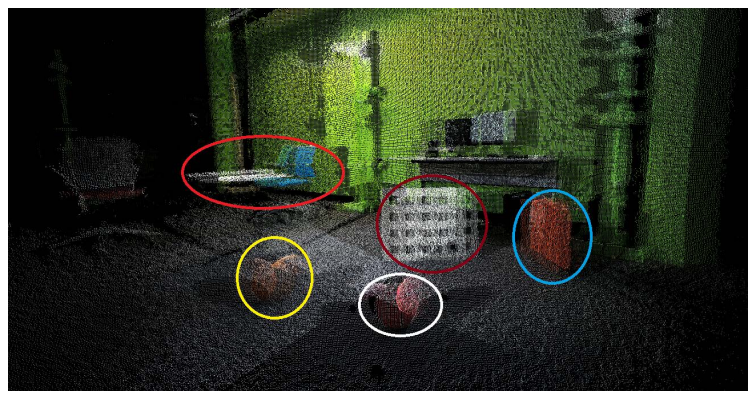

(c)

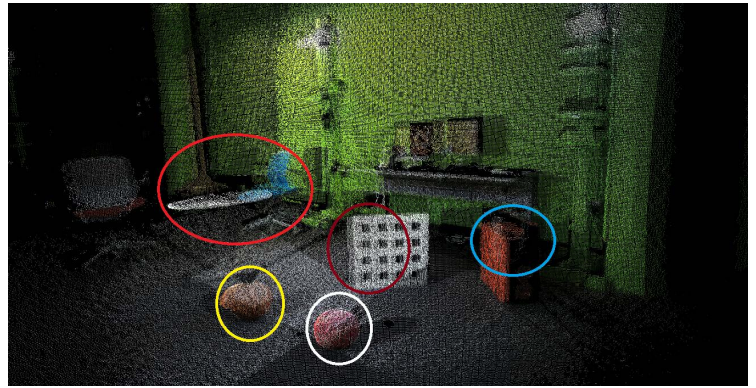

(b)

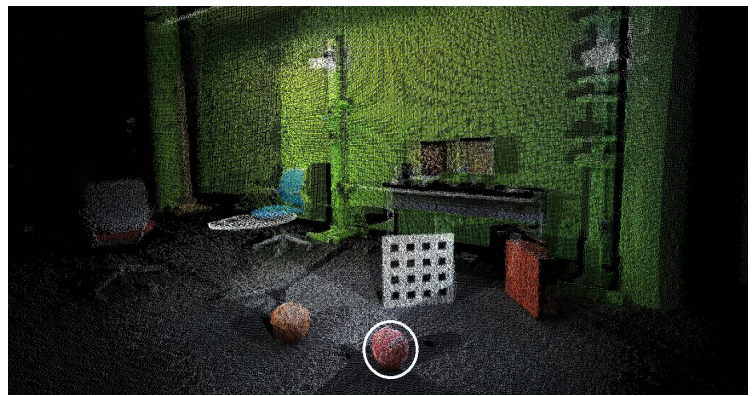

(d)

Figure 6: Calibration results for (a) Checker-board method [36], (b) Plane Intersection approach [3], (c) Visual Pattern approach [17] and (d) Our Skeleton Calibration method. Errors in the calibration results are marked with different colors.

Since all the compared methods use a reference object for calibration, manual intervention is needed to check the calibration errors and re-calibrate. The visual pattern method [17] provides a tool to automatically calibrate the cameras but it still needs the pattern to be placed in such a way that it is visible by all the cameras. This has to be done by stopping the current work and manually placing the reference object in the scene to re-calibrate. In our method, no manual intervention of any kind is needed. The method automatically detects if the calibration is off and re-calibrates the cameras, as long as there is a person in the scene. Since our proposed continuous re-calibration strategy is one of its kind, it can't be evaluated against any of the existing methods.

\subsection{Calibration Propagation}

All methods used assume that the cameras are setup in a closed fashion such that they are all able to see the same scene space. Hence, all camera skeletons are extracted simultaneously. Using this, our method calibrates all the cameras with each other and calibration propagation is not required. In case the capture space is large and has cameras which are not able to see the same person, we need to propagate the calibration values.

Bundle adjustment strategies $[14,21]$ improve the calibration by iteratively reducing the errors in the transformation matrices while performing propagation. When calibration propagation is not required, these strategies can be applied on top of any method to improve calibration results and thus are mainly used for optimization. Bundle adjustment depends directly on the original correspondence points and calibration results. If the calibration is good enough, there might not be much improvement whereas if the original result is poor, bundle adjustment might improve the calibration. This improvement due to bundle adjustment masks the problems existing in the original calibration methods. Hence, we have not applied bundle adjustment on any of the methods and have directly compared the original results so as to have a direct unbiased comparison.

\subsection{Limitations}

Although our skeleton calibration method works significantly better than other methods shown, it is not perfect.

- Our method uses skeleton for obtaining joint correspondence making it limited to only cameras like Kinect that provide skeleton. Use of Kinect was motivated by the fact that large number of researchers rely on the use of multiple Kinects for various projects. Having said that, we are working on a way to eliminate this issue by coming up with a fast and efficient method to obtain feature points similar to the Kinect skeleton joints. These extracted feature points can replace the skeleton, thereby lifting the Kinect dependency.

- The Kinect V2 sensor also seems to have issues estimating the depth of glossy surfaces. Hence, in our method, the person moving in the scene for calibration avoided wearing such clothes (black or shiny).

- Depth estimation in Kinect V2 sensors is done using time of flight. When multiple Kinect V2 sensors are used to capture the same scene, interference is caused between sensors, resulting in noisy depth estimation.

- Accurate depth capture range of Kinect V2 is $0.5-3 m$ [35]. This may not be sufficient for some applications warranting the need for cameras other than Kinect. 


\section{CONCLUSION}

Skeleton-based calibration method was introduced for performing extrinsic calibration of multiple Kinect cameras simultaneously. Our method performs calibration by just using the skeleton information from the captured person. The skeleton is checked for accuracy and a proper joint selection strategy is used for point correspondence. Since there is hardly any occlusion and most of the cameras can simultaneously see the skeleton, calibration propagation or bundle adjustment is not required. Calibration error calculation, being time and processor inexpensive, can be run in the background. This allows for camera misalignment check in real-time, resulting in automatic re-calibration of the cameras. The method is shown to perform well in a scene with 7 Kinect V2 sensors facing each other. The time taken by our method to calibrate the cameras is less than one minute. At the same time the calibration error is very low, the average being $1.5 \mathrm{~cm}$ going to a maximum of $3.5 \mathrm{~cm}$ on rare occasions. Large camera clusters, like the ones used in 3DTI, can be calibrated automatically without any manual intervention, in a few minutes, highly accurately.

\section{ACKNOWLEDGMENTS}

This material is based upon work supported by the National Science Foundation under Grant No. 1012975. Any opinions, findings, conclusions or recommendations expressed in this material are those of author(s) and do not necessarily reflect the views of the National Science Foundation.

\section{REFERENCES}

[1] D. Alexiadis, D. Zarpalas, and P. Daras. Fast and smooth 3d reconstruction using multiple rgb-depth sensors. In Visual Communications and Image Processing Conference, 2014 IEEE, pages 173-176, Dec 2014.

[2] K. S. Arun, T. S. Huang, and S. D. Blostein. Least-squares fitting of two 3-d point sets. IEEE Trans. Pattern Anal. Mach. Intell., 9(5):698-700, May 1987.

[3] E. Auvinet, J. Meunier, and F. Multon. Multiple depth cameras calibration and body volume reconstruction for gait analysis. In Information Science, Signal Processing and their Applications (ISSPA), 2012 11th International Conference on, pages 478-483, July 2012.

[4] S. Beck and B. Froehlich. Volumetric calibration and registration of multiple rgbd-sensors into a joint coordinate system. In 3D User Interfaces (3DUI), 2015 IEEE Symposium on, pages 89-96. IEEE, 2015.

[5] M. Carraro, M. Munaro, A. Roitberg, and E. Menegatti. Improved Skeleton Es timation by Means of Depth Data Fusion from Multiple Depth Cameras, pages 1155-1167. Springer International Publishing, Cham, 2017.

[6] C. H. Chen, J. Favre, G. Kurillo, T. P. Andriacchi, R. Bajcsy, and R. Chellappa. Camera networks for healthcare, teleimmersion, and surveillance. Computer, 47(5):26-36, May 2014

[7] S. Chen, Z. Gao, K. Nahrstedt, and I. Gupta. 3dti amphitheater: Towards 3dti broadcasting. ACM Trans. Multimedia Comput. Commun. Appl., 11(2s):47:1-47:22, Feb. 2015.

[8] L. Cruz, D. Lucio, and L. Velho. Kinect and rgbd images: Challenges and applications. In Graphics, Patterns and Images Tutorials (SIBGRAPI-T), 2012 25th SIBGRAPI Conference on, pages 36-49, Aug 2012.

[9] K. Desai, K. Bahirat, S. Ramalingam, B. Prabhakaran, T. Annaswamy, and U. E. Makris. Augmented reality-based exergames for rehabilitation. In Proceedings of the 7th International Conference on Multimedia Systems, MMSys '16, pages 22:1-22:10, 2016.

[10] K. Desai, U. H. H. Belmonte, R. Jin, B. Prabhakaran, P. Diehl, V. A. Ramirez, V. Johnson, and M. Gans. Experiences with multi-modal collaborative virtual laboratory (mmcvl). In 2017 IEEE Third International Conference on Multimedia Big Data (BigMM), pages 376-383, April 2017.

[11] K. Desai, S. Raghuraman, R. Jin, and B. Prabhakaran. Qoe studies on interactive 3d tele-immersion. In 2017 IEEE International Symposium on Multimedia (ISM), Dec 2017.

[12] D. Dwarakanath, C. Griwodz, P. Halvorsen, and J. Lildballe. Study the effects of camera misalignment on 3d measurements for efficient design of vision-based inspection systems. In A. Likas, K. Blekas, and D. Kalles, editors, Artificial
Intelligence: Methods and Applications, pages 150-163, Cham, 2014. Springer International Publishing.

[13] T. Elgamal and K. Nahrstedt. Multicamera summarization of rehabilitation sessions in home environment. In Proceedings of the 2017 ACM on Multimedia Conference, MM '17, pages 1381-1389, 2017.

[14] Y. Furukawa and J. Ponce. Accurate camera calibration from multi-view stereo and bundle adjustment. International fournal of Computer Vision, 84(3):257-268, Sep 2009.

[15] D. Herrera, J. Kannala, and J. Heikkilä. Joint depth and color camera calibration with distortion correction. IEEE Transactions on Pattern Analysis and Machine Intelligence, 34(10):2058-2064, 2012.

[16] B. K. P. Horn, H. Hilden, and S. Negahdaripour. Closed-form solution of absolute orientation using orthonormal matrices. FOURNAL OF THE OPTICAL SOCIETY AMERICA, 5(7):1127-1135, 1988.

[17] M. Kowalski, J. Naruniec, and M. Daniluk. Livescan3d: A fast and inexpensive 3d data acquisition system for multiple kinect v2 sensors. In 3D Vision (3DV), 2015 International Conference on, pages 318-325, Oct 2015.

[18] G. Kurillo and R. Bajcsy. 3d teleimmersion for collaboration and interaction of geographically distributed users. Virtual Reality, 17(1):29-43, 2013.

[19] B. Li, L. Heng, K. Koser, and M. Pollefeys. A multiple-camera system calibration toolbox using a feature descriptor-based calibration pattern. In 2013 IEEE/RSf International Conference on Intelligent Robots and Systems, pages 1301-1307. IEEE, 2013.

[20] M. A. Livingston, J. Sebastian, Z. Ai, and J. W. Decker. Performance measurements for the microsoft kinect skeleton. In 2012 IEEE Virtual Reality Workshops (VRW), pages 119-120, March 2012.

[21] M. I. A. Lourakis and A. A. Argyros. Sba: A software package for generic sparse bundle adjustment. ACM Trans. Math. Softw., 36(1):2:1-2:30, Mar. 2009.

[22] A. Maimone, J. Bidwell, K. Peng, and H. Fuchs. Enhanced personal autostereoscopic telepresence system using commodity depth cameras. Computers \& Graphics, 36(7):791-807, 2012.

[23] R. Mekuria, M. Sanna, E. Izquierdo, D. Bulterman, and P. Cesar. Enabling geometry-based 3-d tele-immersion with fast mesh compression and linear rateless coding. Multimedia, IEEE Transactions on, 16(7):1809-1820, Nov 2014.

[24] K. A. F. Mora and J. M. Odobez. Gaze estimation from multimodal kinect data. In 2012 IEEE Computer Society Conference on Computer Vision and Pattern Recognition Workshops, pages 25-30, June 2012.

[25] M. Munaro, F. Basso, and E. Menegatti. Openptrack: Open source multi-camera calibration and people tracking for rgb-d camera networks. Robotics and $\mathrm{Au}$ tonomous Systems, 75(Part B):525 - 538, 2016.

[26] J. C. Núñez, R. Cabido, A. S. Montemayor, and J. J. Pantrigo. Real-time human body tracking based on data fusion from multiple rgb-d sensors. Multimedia Tools Appl., 76(3):4249-4271, Feb. 2017.

[27] S. Obdrzálek, G. Kurillo, F. Ofli, R. Bajcsy, E. Seto, H. Jimison, and M. Pavel. Accuracy and robustness of kinect pose estimation in the context of coaching of elderly population. In 2012 Annual International Conference of the IEEE Engineering in Medicine and Biology Society, pages 1188-1193, Aug 2012.

[28] M. Ruan and D. Huber. Calibration of 3d sensors using a spherical target. In 2014 2nd International Conference on 3D Vision, volume 1, pages 187-193, Dec 2014.

[29] T. Schöps, J. L. Schönberger, S. Galliani, T. Sattler, K. Schindler, M. Pollefeys, and A. Geiger. A multi-view stereo benchmark with high-resolution images and multi-camera videos. In 2017 IEEE Conference on Computer Vision and Pattern Recognition (CVPR), pages 2538-2547, July 2017.

[30] J. Shotton, A. Fitzgibbon, M. Cook, T. Sharp, M. Finocchio, R. Moore, A. Kipman, and A. Blake. Real-time human pose recognition in parts from single depth images. In R. Cipolla, S. Battiato, and G. M. Farinella, editors, Machine Learning for Computer Vision, volume 411 of Studies in Computational Intelligence, pages 119-135. Springer Berlin Heidelberg, 2013.

[31] A. Staranowicz, G. R. Brown, F. Morbidi, and G. L. Mariottini. Easy-to-use and accurate calibration of rgb-d cameras from spheres. In Pacific-Rim Symposium on Image and Video Technology, pages 265-278. Springer, 2013.

[32] C. Strecha, W. von Hansen, L. V. Gool, P. Fua, and U. Thoennessen. On benchmarking camera calibration and multi-view stereo for high resolution imagery. In 2008 IEEE Conference on Computer Vision and Pattern Recognition, pages 1-8, June 2008.

[33] P. C. Su, J. Shen, and M. U. Rafique. Rgb-d camera network calibration and streaming for 3d telepresence in large environment. In 2017 IEEE Third International Conference on Multimedia Big Data (BigMM), pages 362-369, April 2017.

[34] S. Umeyama. Least-squares estimation of transformation parameters between two point patterns. IEEE Trans. Pattern Anal. Mach. Intell., 13(4):376-380, Apr. 1991.

[35] L. Yang, L. Zhang, H. Dong, A. Alelaiwi, and A. El Saddik. Evaluating and improving the depth accuracy of kinect for windows v2. Sensors fournal, IEEE, 15(8):4275-4285, Aug 2015.

[36] Z. Zhang. A flexible new technique for camera calibration. IEEE Trans. Pattern Anal. Mach. Intell., 22(11):1330-1334, Nov. 2000. 